\title{
Proof of Credibility: A Blockchain Approach for Detecting and Blocking Fake News in Social Networks
}

\author{
Mohamed Torky ${ }^{1}$ \\ Information Technology Department \\ Faculty of Computer and Information System \\ Islamic University of Madinah \\ Madinah, Saudi Arabia \\ Higher Institute of Computer Science and Information \\ Systems \\ Culture and Science City Academy \\ 6 October-Giza-Egypt \\ Scientific Research Group in Egypt (SRGE) \\ ORCID No: 0000-0002-3229-9794
}

\author{
Emad Nabil ${ }^{2}$ \\ Computer Science Department \\ Faculty of Computer and Information System, Islamic \\ University of Madinah, Madinah, Saudi Arabia \\ Faculty of Computers and Artificial Intelligence \\ Cairo University, Giza, Egypt \\ Wael Said ${ }^{3}$ \\ Computer Science Department, Faculty of Computers and \\ Informatics, Zagazig University, Zagazig, Egypt
}

\begin{abstract}
Rumors and misleading information detection and prevention still represent a big challenge against social network developers and researchers. Since newsworthy information propagation is a traditional behavior of most of the users in social media, then verifying information credibility and reliability is indeed a vital security requirement for social network platforms. Due to its immutability, security, tamperproof and P2P design, Blockchain as a powerful technology can provide a magical solution to overcome this challenge. This Paper introduces a novel blockchain approach called Proof of Credibility $(\mathrm{PoC})$ for detecting fake news and blocking its propagation in social networks. The functionality of the PoC protocol has been simulated on two datasets of newsworthy tweets collected from different news sources on Twitter. The results clarified a satisfying performance and efficiency of the proposed approach in detecting rumors and blocking its propagation.
\end{abstract}

Keywords-Blockchain technology; social networks; fake news detection

\section{INTRODUCTION}

Sharing newsworthy information on social media platforms like Facebook, Twitter, and Instagram without evaluating its credibility has popped out a big challenge. Disseminating Misleading information and fake news across social networks represent a big dilemma against researchers and Social Network Service Providers (SNPs) [1]. The political elections are the best witness to sharing a lot of rumors patterns across social media. For instance, more than 1,000 reports on politics and election were declared as fake in the Jakarta Gubernatorial Election in 2017 [2]. Also, Fake news and misleading information can misuse the reputation of countries as well as jeopardize international relations. In 2017, the Twitter account of Qatar's state news agency had been penetrated and published false news to criticize aspects of the Arab Gulf and US foreign policy towards Iran. The neighbor countries like UAE, Bahrain, Saudi Arabia, and Egypt broke diplomatic ties with Qatar [3]. With advances in AI technologies like Machine learning [4], deep learning [5] and digital animations [6], developing malicious social bots for propagating fake news represents a big challenge. According to Gartner's predictions for 2018, most of the people will read and believe more false news than real news by 2020 [7]. Although AI has the best opportunity of recognizing misleading information, it is seriously able to create fake content on social media. Unfortunately, recently, the power of AI algorithms for identifying fake news is lower than its ability to create it [8]. Therefore, it has become an essential need to search for an alternative robust and efficient technology for handling this problem. Developing blockchain-based solutions have the potential to change the way of information creation and propagation [9]. Blockchain can provide trustable, immutable, verifiable, reliable, and transparent transactions for designing a trusted social system [10]. In this paper, we investigate the possibility of using blockchain theory for developing a novel protocol called Proof of Credibility (PoC) able to verify shared information and detect misleading information across social networks. The proposed protocol is simulated on 1003 newsworthy tweets around the trending topic \#ISIS, and 802 newsworthy tweets around the trending topic \#Halamadrid which are collected from different news sources on Twitter. The results clarified the possibility of developing a standard blockchain consensus for verifying information credibility as well as blocking rumor propagation across social networks. PoC will represent the first blockchain protocol that can be utilized for solving the problem of fake news and misleading information in social networks. The rest of this paper can be organized as follows: Section 2 presents the literature review. Section 3 discusses the proposed blockchain protocol. Section 4 presents the simulation results. Finally, Section 5 presents the conclusion and future work. 


\section{LITERATURE REVIEW}

There is a relatively small body of literature that is concerned with using blockchain as a solution tool for solving social network security problems. Moreover, unfortunately, there are relatively few historical blockchain approaches utilized for solving fake news and misleading information problem specifically. However, the literature introduced some blockchain techniques for handling specified problems in social networks. For example, A blockchain is configured as a data bank accessible by social network nodes based on rules and preferences of the nodes upon authorization by the blockchain configured data bank for facilitating social integrity networking [11]. Another work in investigating some of the recent blockchain platforms to determine if they fulfill Metcalfe's Law for modeling social media networks based on digital currency protocols [12]. The authors in [13] investigate the potential of utilizing the U-share-blockchain mechanism to enable users to manage and track all posted contents they share over the social network. Yahiatene et al. in [14] introduced a novel framework that depends on two main components: Software-Defined Vehicular Networks (SDVN) and Blockchain for authenticating the transactions and providing anonymity of data in a distributed manner across the social network. Moreover, blockchain-based social networks can be utilized for combining fog computing to develop security architecture for managing IoT systems by creating tamperproof digital identities in a trustless domain [15]. An additional blockchain contribution to social networks is Tweet-chain [16]. Tweet-chain is a novel social network model-based on blockchain for managing public posts based on Proof of Concept protocol [17] to ensure social transaction security instead of proof of work. It can work without the required trustworthiness assumption for the social network provider. Fakechain [18] is another blockchain consensus -based on data mining algorithms for authenticating shared information [19] across the social network and detecting fake news [20]. Detecting fake news can be achieved by utilizing the functionality of Ethereum blockchain combined with the Breadth-First Search (BFS) algorithm [21].

\section{Proof OF CREDibiLITy (PoC) APPROACH}

Automatic rumors recognition and blocking it propagation are indeed very hard challenges to design trusted social networks. There are a number of techniques that have been developed to fight against misleading information in social networks [22][23]. The recent researches utilize the benefits of Artificial Intelligence (AI)-based techniques for detecting misleading information [24] [25]. Other methods have been based on sentiment analysis combined with IP network analysis [26] for detecting social network rumors. Moreover, some studies tried to solve this problem based on Natural Language Processing techniques [27]. However, these studies have a lot of limitations and inaccurate results in detecting fake news. For example, while AI-based techniques can help in limiting the rise of misleading information, it is not invincible. Such that, It may occasionally make a lot of False Positive and False Negative errors. So, Social network systems can be redesigned with blockchain to give control of the shared posts back to the users themselves in $\mathrm{P} 2 \mathrm{P}$ communication. The major advantages of utilizing blockchain-based techniques for detecting misleading information are:

1) Better privacy and protection.

2) Decentralized social network architecture.

3) User control based on a distributed content.

4) Online automatic verification for shared information.

5) Grants a robust authentication and ensures anonymity.

In this paper, we propose a novel Blockchain algorithm called Proof of Credibility (PoC) for detecting and blocking fake news in social networks. The idea behind $\mathrm{PoC}$ is that reengineering the social networks as decentralized networks, where users are represented as peers. Each peer shares a distributed ledger, which represented as an immutable, cryptographically secured record of detected rumors. The distributed ledger is designed as a sequence of blocks, where each block in the blockchain represents a new detected number of rumors. For example, a new block is added to the chain if the blockchain system detected 10 rumors. Moreover, The detection functionality is executed by PoC chain code which is shared also with all peers in the social network platform. They can execute POC chain code via a blockchain browser. "Fig. 1" shows the conceptual view of the blockchain-based social network system, and Fig. 2 explains the design model of the PoC protocol.

With PoC -based social network systems there is no need for the third party to verify the shared information and identifying rumors, such that peers will do this task based on the PoC blockchain system. Each block in the blockchain involves a fixed number of fake news. This number is agreed by the peers of social networks and is defined in the PoC consensus. For example, a new block is added to the chain after identifying 100 rumors in the social networking system.

The idea behind PoC is evaluating the credibility of the source who shared the information. As we interested in newsworthy information, the source may be an online newspaper, magazine, TV-News channel, Radio, Wire services, or a blog by users. The functionality of $\mathrm{PoC}$ is based on the Best Match technique (BM25F) metric [28]. It can be used for evaluating the credibility of online sources of newsworthy information [29].

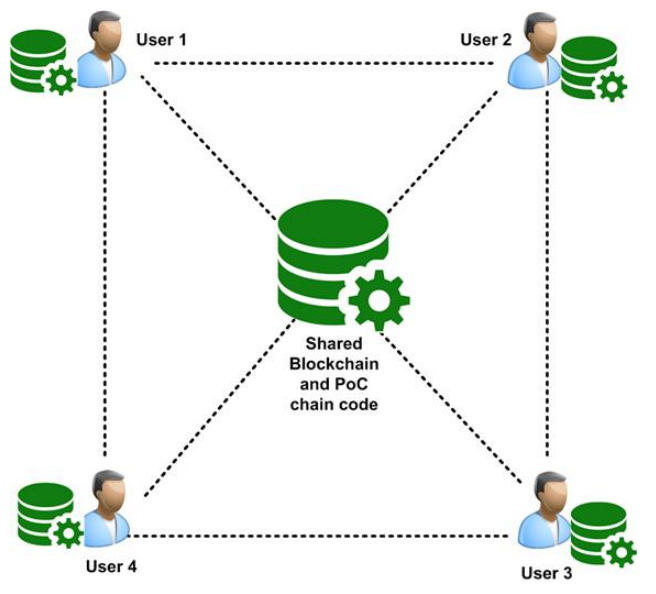

Fig. 1. Conceptual view of the Blockchain-based Social Network System. 


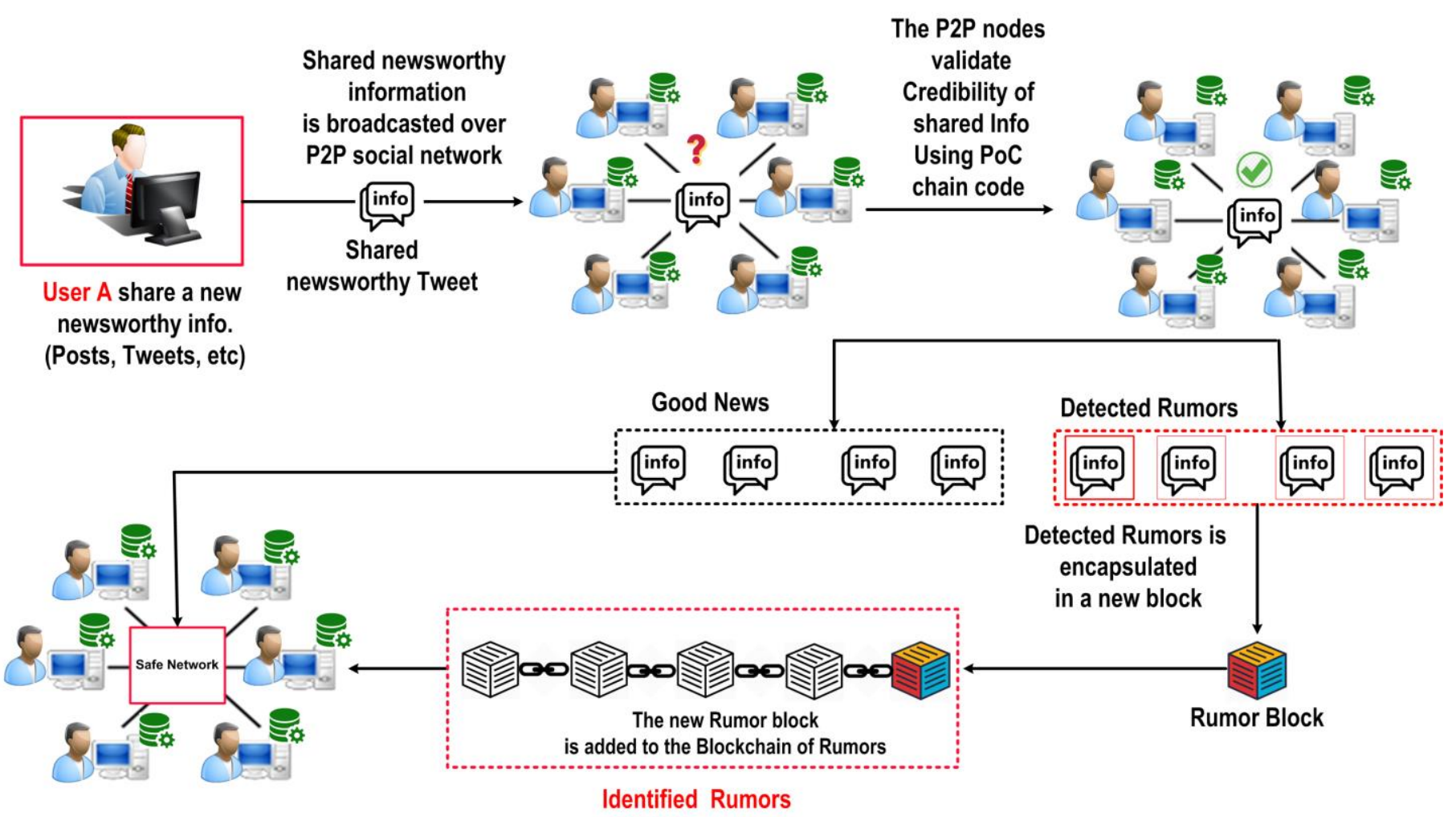

Fig. 2. Proof of Credibility (PoC) Design Model.

Source credibility can be calculated based on two dependent factors. The first factor is $W(S i)$, which is used for producing the credibility weight (W) value to Information Sources as formulated in equation (1).

$W\left(S_{i}\right)=\frac{\text { Occure } \times \text { Boost }}{\left[(1-b)+b \times \frac{\text { \#Posts }}{\text { \#Followers }}\right]}$

Where occur is the number of posts/ tweets that are associated with the source $S_{i}$ in all shared Posts/Tweets of a specific trending topic in a specific time $t$. Boost is called boost factor, which applied according to the type of the source of information as presented in Table I, the boost values are taken from News Trust [30] which is a review tool rate the news based on journalistic quality, not just popularity. So, the handled information must be classified according to the source type to assign the appropriate boost value from Table I. $b$ is a constant, such that $0.5<\mathrm{b}<0.8$. \#Posts is the number of all posts that are shared by the source $S_{i}$. \#followers is number of followers who follow the source $S_{i}$. The second factor is Logarithmic Ratio (LR) of the number of posts/tweets in the collection (i.e. the number of shared information within a specific period defined in $\mathrm{PoC}$ protocol) that share the same source of news as in equation (2).

$\operatorname{LR}\left(S_{i}\right)=\log \frac{N+n\left(S_{i}\right)+0.5}{n\left(S_{i}\right)+0.5}$
Where $N$ is the number of posts in the collection and $n\left(S_{i}\right)$ is the number of posts, which are associated with $\left(S_{i}\right)$. Finally, the Source Credibility (SC) value can be calculated as in equation (3).

$S C\left(S_{i}\right)=\frac{W\left(S_{i}\right)}{K_{1}+W\left(S_{i}\right)} \times \operatorname{LR}\left(S_{i}\right)$

Where, $K_{l}$ is a constant value used as a free Parameter, such that $1.2<\mathrm{K}_{1}<2$.

TABLE. I. Boost VALUES OF THE Most COMMON SOURCES OF NEWSWORTHY INFORMATION [30]

\begin{tabular}{|l|l|}
\hline News Source & Boost values \\
\hline Newspapers & 3.29 \\
\hline Magazines & 3.68 \\
\hline TV-News Channels & 3.54 \\
\hline Online Sites & 3.69 \\
\hline Radio & 3.60 \\
\hline Wire Service & 3.48 \\
\hline Blogs & 3.55 \\
\hline
\end{tabular}


The PoC-chain code which implements the detection methodology and evaluates information credibility can be implemented as in Algorithm 1.

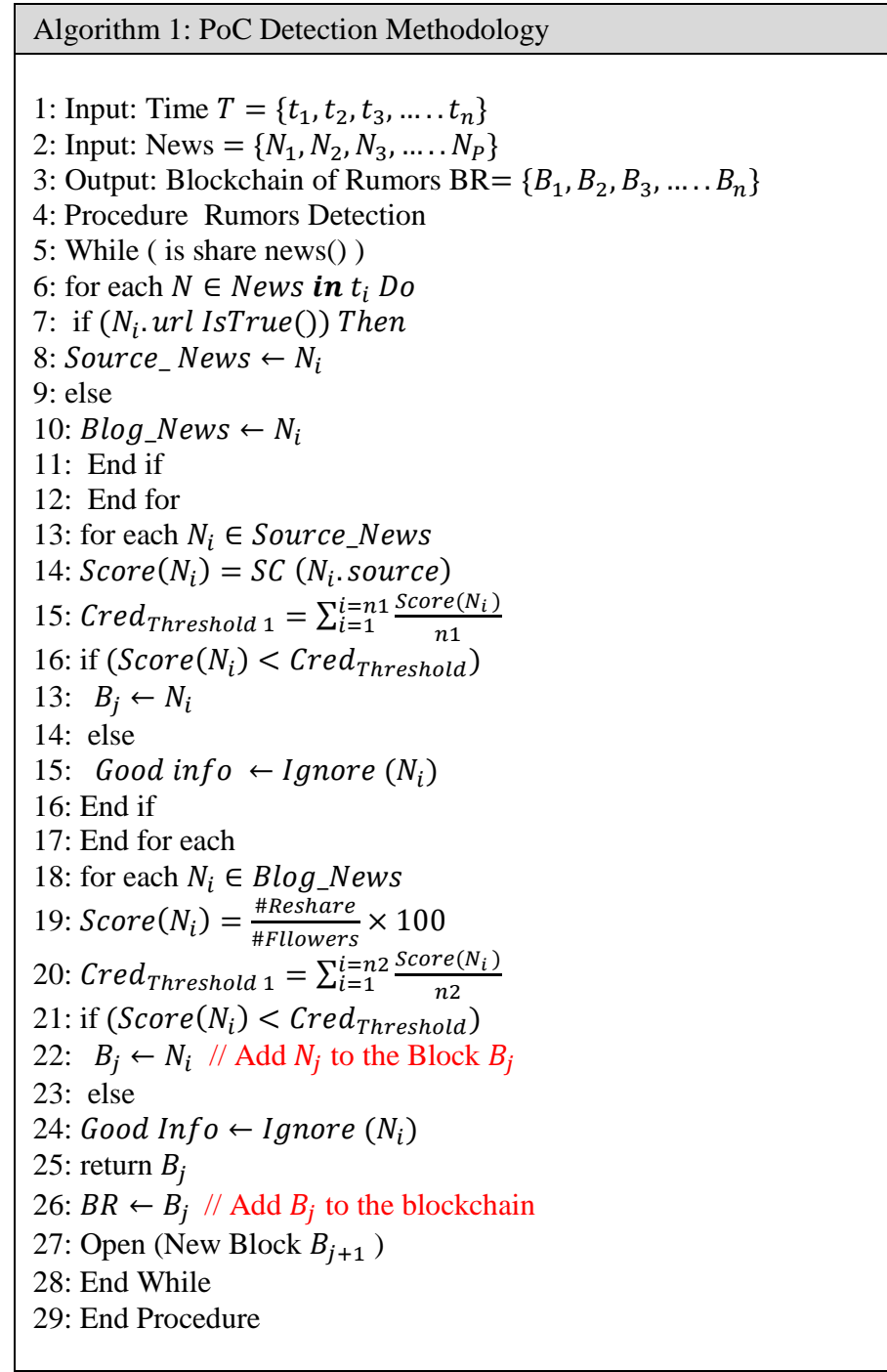

\section{SimULATION RESULTS}

For investigating the realism and effectiveness of the proposed $\mathrm{PoC}$ approach, a simulation experiment has been applied in two datasets collected from Twitter. The two datasets are described as two trending topics (\#ISIS, and \#Halamadrid) that involve several newsworthy tweets that have been tweeted by multiples sources of information. The Twitter $\mathrm{R}$ library tool [31] has been used as a software tool for collecting tweets of each trending topic across four-time intervals. The general description of the two datasets can be summarized in Table II. The simulation results of applying the PoC algorithm on the two mentioned datasets show that the PoC algorithm could detect the fake news in the two mentioned datasets as follows, 215 tweets in block 1, 284 tweets in block two, 224 in block three, and 208 in block four as depicted in Table III. Fig. 3 depicts also the total numbers of fake news for the seven sources types of information. The results show that online newspapers are the most source type of fake news in the
\#ISIS dataset, while Wire Service is the most source type of fake news in the \#Halamadrid dataset. The pi plot in Fig. 4 depicts the percentages of detected fake news in the two studied datasets.

For verifying this obtained results, the set of detected fake news (i.e.931 tweets) and good news (i.e. 874 tweets) are outsourced to the Xpertin platform [32]. It has a set of the best experts in social media services. The verification results and feedback of Xpertin reported that only $91 \%$ of detected fake news is indeed fake (i.e. only 847 tweets are fake news) and $86 \%$ of good news is indeed good (i.e. only 752 tweets are good news). According to Xpertin's feedback, this result means that the PoC technique failed to recognize 84 tweets as fake tweets and 57 tweets as good tweets. So, according to True Positive (TP), False Positive (FP), True Negative (TN) and False Negative (FN) values, Precision, Recall, and Accuracy can be calculated as in equations $4,5,6$. Fig. 5 presents the Precision, Recall, and Accuracy results of the PoC technique.

Precision $=\frac{T P}{T P+F P}$

Such that $T P$ is the number of True Positive tweets and $F P$ is the number of False Positive tweets.

Recall $=\frac{T P}{T P+F N}$

Such that $T P$ is the number of True Positive tweets and $F N$ is the number of False Negative tweets

Accuracy $=\frac{T P+T N}{P+N}$

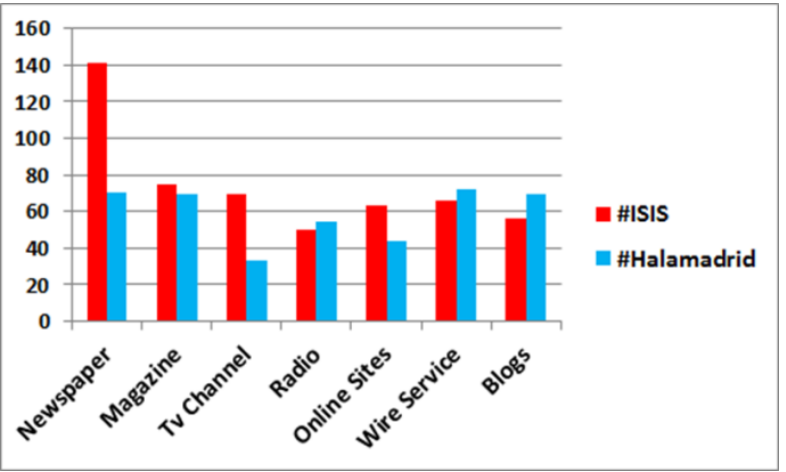

Fig. 3. The Total Numbers of Fake News for the Seven Sources Types of Information in the Two Studied Datasets after Constituting Four Blocks in the Blockchain.

Percentages of fake news in the two datasets

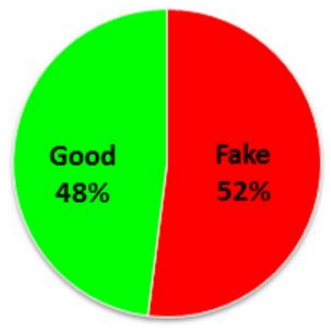

Fig. 4. The Total Percentages of Detected Fake News in the Two Data Sets. 
TABLE. II. Two DATASETS DESCRIPTION, \#ISIS, AND \# HALAMADRID

\begin{tabular}{|c|c|c|c|c|c|c|c|c|c|c|}
\hline \multirow[b]{2}{*}{ Source } & \multicolumn{5}{|l|}{ \#ISIS } & \multicolumn{5}{|c|}{ \#Halamadrid } \\
\hline & $\begin{array}{l}\# \\
\text { Tweets }\end{array}$ & t1 & t2 & t3 & t4 & \# Tweets & t1 & t2 & $\mathbf{t 3}$ & $\mathbf{t 4}$ \\
\hline Newspapers & 281 & 66 & 73 & 59 & 83 & 183 & 39 & 46 & 57 & 41 \\
\hline Magazines & 149 & 46 & 34 & 38 & 31 & 156 & 47 & 28 & 37 & 44 \\
\hline TV Channels & 132 & 36 & 29 & 43 & 24 & 108 & 25 & 22 & 43 & 18 \\
\hline Radios & 99 & 25 & 16 & 22 & 36 & 101 & 29 & 33 & 17 & 22 \\
\hline Online Sites & 114 & 22 & 35 & 30 & 27 & 97 & 16 & 26 & 19 & 36 \\
\hline Wire Service & 109 & 26 & 38 & 26 & 19 & 50 & 14 & 15 & 10 & 11 \\
\hline Blogs & 119 & 25 & 23 & 39 & 32 & 107 & 30 & 22 & 26 & 29 \\
\hline Sum & 1003 & 246 & 248 & 257 & 252 & 802 & 200 & 192 & 209 & 201 \\
\hline
\end{tabular}

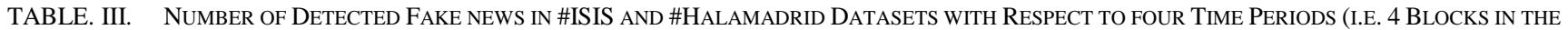
BLOCKCHAIN) AND SEVEN SOURCES

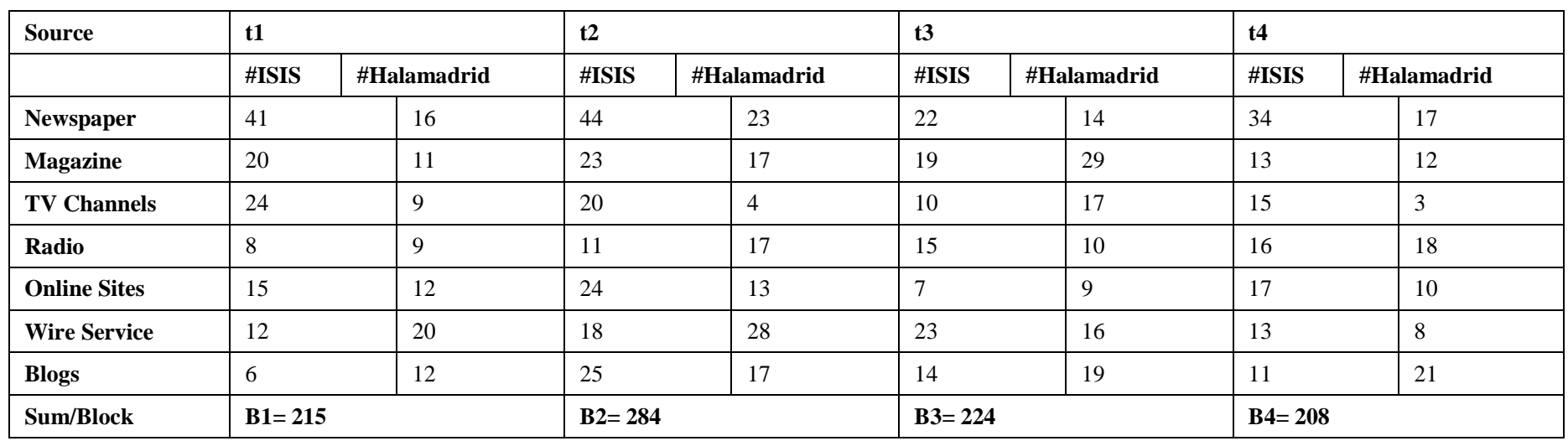

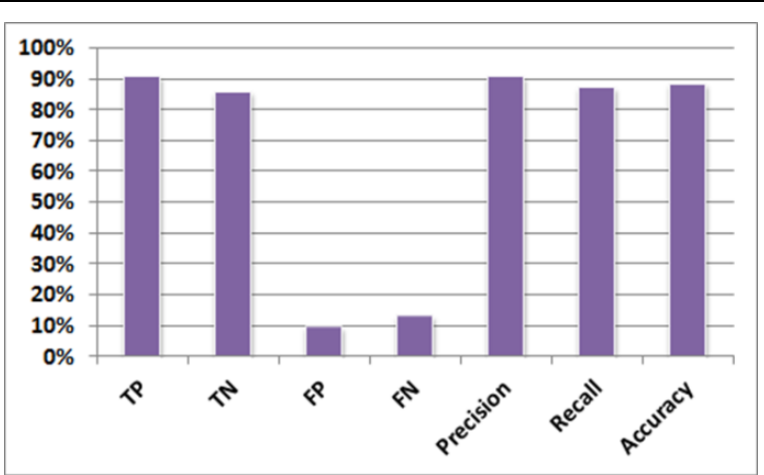

Fig. 5. TP, TN, FP, FN, Precision, Recall, Accuracy Results.

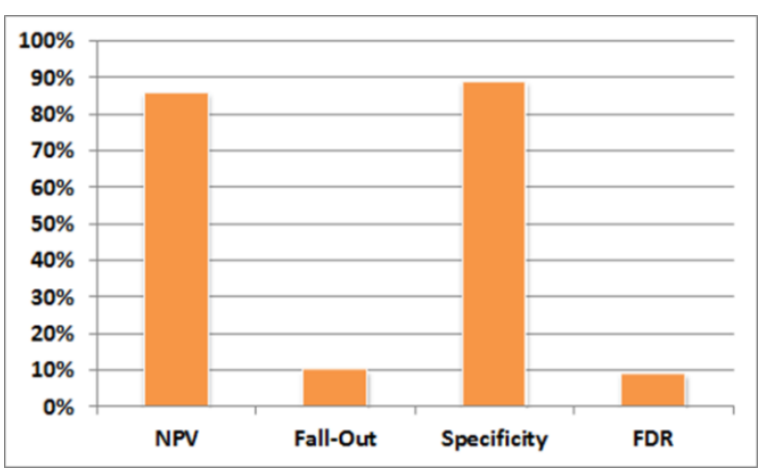

Fig. 6. NPV, Fall-Out, Specificity, and FDR Results.
Such that $\mathrm{P}=(\mathrm{TP}+\mathrm{FN})$, and $\mathrm{N}=(\mathrm{FP}+\mathrm{TN})$ and $\mathrm{TN}$ is the True Negative tweets.

Moreover, the Negative Predictive Value (NPV), Fall-Out, Specificity, and False Discovery Rate (FDR) are evaluated for PoC mechanism as in equations 7, 8, 9, 10 and the results depicted in Fig. 6.

$N P V=\frac{T N}{T N+F N}$

Fall - Out $=\frac{F P}{F P+T N}$

Specificity $=\frac{T N}{T N+F P}$

$F D R=\frac{F P}{F P+T P}$

\section{DISCUSSION}

Prior studies that have noted the importance of utilizing blockchain for detecting and preventing fake news in social networks didn't introduce a novel and real blockchain technique for solving this problem [19-23]. These studies were limited to only present some state of the art about the problem without proposing a novel blockchain-based algorithm able to detect fake news in social networks. This study set out with the aim of proposing the first blockchain consensus called Proof of Credibility (PoC) for detecting and preventing fake news and rumors in social networks. The most interesting finding of this study is the satisfied value of accuracy (89\%) in detecting fake 
news although the proposed algorithm is an initial blockchain algorithm that may require more functions and metrics for future improvements. Other interesting findings are the low values of Fall-Out and False Discovery Rate (FDR) which are $10 \%$ and $9 \%$ respectively in recognizing rumors and Fake news. It is somewhat surprising that about $52 \%$ of the total number of tweets in the two-handled datasets is recognized as fake news. This finding was unexpected and suggests that it should be there are more considerations to additional features ( such as consistency and coherency of news, and general commons and acceptability [33]) of handled online newsworthy information for assessing its credibility in more accuracy. However, these findings support and encourage utilizing blockchain algorithms in managing and controlling the information propagation in social networks. As this result has not previously been described, and all previous studies handled the problem as jus as a theoretical investigation and review of the literature [19-23], so there are no comparison factors we can investigate. However, this study introduces the first real blockchain algorithm that can be compared with other blockchain-based algorithms in detecting fake news in social networks, which can be developed in the future. These findings must be interpreted with caution because the proposed algorithm is simulated on a limited amount of newsworthy tweets collected from two trending topics on twitter. For more accurate analysis and evaluation, the proposed algorithm should be improved and applied on benchmark and standardized datasets and should be applied within more periods of time for investigating the growth of blockchain of rumors in more tracing. However, this finding, while preliminary, suggests utilizing the first blockchain-based algorithm for managing and controlling information dissemination in social networks in order to detect fake news and misleading information. Improving the proposed algorithm with more measures of information credibility and simulating its functionality in benchmark and standardized datasets are more important issues for future research.

\section{CONCLUSION}

The aim of the present research was to examine utilizing a novel blockchain-based algorithm for detecting and preventing fake news and misleading information across social networks. The study proposed a novel blockchain consensus called Proof of Credibility (PoC) for detecting fake news in social media platforms. The study has shown satisfying preliminary results obtained from simulating the proposed algorithm on two trending topics from Twitter. The experimental results have clarified the effectiveness of PoC in detecting Fake news with an accuracy of about $89 \%$, Fall-Out, and False Discovery Rate about $10 \%$ and $9 \%$ respectively. An implication of these results is the possibility of proposing additional blockchain algorithms for managing and controlling online information dissemination across social networks and can be compared with the proposed one. Before this study, the idea of utilizing blockchain for detecting fake news was purely anecdotal. This study was limited by the absence of a benchmark and standardized datasets for investigating $\mathrm{PoC}$ efficiency in more accuracy. However, this study introduces valuable insight into leveraging Blockchain and developing novel consensuses for more managing and controlling rumors propagation in social networks. More research using benchmark datasets and controlled trials is needed for improving PoC functionality and efficiency in recognizing rumors and fake news in the next studies.

\section{REFERENCES}

[1] Vosoughi S, Roy D, Aral S. The spread of true and false news online. Science. 2018 Mar 9;359(6380):1146-51.

[2] BBC News, How fake news and hoaxes have tried to derail Jakarta's election, [online], available, https://www.bbc.com/news/world-asia39176350 (accessed 5 Oct. 2019).

[3] Robert W and William M. A, Who Planted the Fake News at Center of Qatar Crisis?, [online], available, https://www.nbcnews.com/news/ world/who-planted-fake-news-center-qatar-crisis-n784056 (accessed 5 Oct. 2019).

[4] Kumar A, Sangwan SR. Rumor Detection Using Machine Learning Techniques on Social Media. International Conference on Innovative Computing and Communications 2019 (pp. 213-221). Springer, Singapore.

[5] Gong Q, Chen Y, He X, Zhuang Z, Wang T, Huang H, Wang X, Fu X. DeepScan: Exploiting deep learning for malicious account detection in location-based social networks. IEEE Communications Magazine. 2018 Nov 19;56(11):21-7.

[6] Hou R, Pérez-Rosas V, Loeb S, Mihalcea R. Towards Automatic Detection of Misinformation in Online Medical Videos. arXiv preprint arXiv:1909.01543. 2019 Sep 4.

[7] Gartner, "Gartner Top Strategic Predictions for 2018 and Beyond", 2017.Available: https://www.gartner.com/smarterwithgartner/gartnertopstrategic-predictions-for-2018-and-beyond/ [Accessed 6 Oct. 2019].

[8] Paschen J. Investigating the emotional appeal of fake news using artificial intelligence and human contributions. Journal of Product \& Brand Management. 2019 May 6.

[9] Tee WJ, Murugesan RK. Trust Network, Blockchain and Evolution in Social Media to Build Trust and Prevent Fake News. In2018 Fourth International Conference on Advances in Computing, Communication \& Automation (ICACCA) 2018 Oct 26 (pp. 1-6). IEEE.

[10] Chen Y, Li Q, Wang H. Towards trusted social networks with blockchain technology. arXiv preprint arXiv:1801.02796. 2018 Jan 9.

[11] Shah SN, inventor; Netspective Communications LLC, assignee. Device-driven non-intermediated blockchain system over a social integrity network. The United States patent application US 15/372,699. 2017 Mar 30.

[12] Alabi K. Digital blockchain networks appear to be following Metcalfe's Law. Electronic Commerce Research and Applications. $2017 \mathrm{Jul}$ $1 ; 24: 23-9$.

[13] Chakravorty A, Rong C. U-share: user-controlled social media based on blockchain. InProceedings of the 11th international conference on ubiquitous information management and communication 2017 Jan 5 (p. 99). ACM.

[14] Yahiatene Y, Rachedi A. Towards a blockchain and software-defined vehicular network approaches to secure vehicular social network. In2018 IEEE Conference on Standards for Communications and Networking (CSCN) 2018 Oct 29 (pp. 1-7). IEEE.

[15] Zhu X, Badr Y. Fog Computing Security Architecture for the Internet of Things using Blockchain-based Social Networks. In2018 IEEE International Conference on the Internet of Things (iThings) and IEEE Green Computing and Communications (GreenCom) and IEEE Cyber, Physical and Social Computing (CPSCom) and IEEE Smart Data (SmartData) 2018 Jul 30 (pp. 1361-1366). IEEE.

[16] Buccafurri F, Lax G, Nicolazzo S, Nocera A. Tweetchain: An alternative to blockchain for crowd-based applications. In International Conference on Web Engineering 2017 Jun 5 (pp. 386-393). Springer, Cham.

[17] Song G, Kim S, Hwang H, Lee K. Blockchain-based Notarization for Social Media. In2019 IEEE International Conference on Consumer Electronics (ICCE) 2019 Jan 11 (pp. 1-2). IEEE.

[18] Gomes AJ, Fernandes AM, Leithardt VR. FakeChain: A Blockchain Architecture to Ensure Trust in Social Media Networks. In Quality of Information and Communications Technology: 12th International 
Conference, AQUATIC 2019, Ciudad Real, Spain, September 11-13, 2019, Proceedings (p. 105). Springer Nature.

[19] Huckle S, White M. Fake news: a technological approach to proving the origins of content, using blockchains. Big data. 2017 Dec 1;5(4):356-71.

[20] Tee WJ, Murugesan RK. Trust Network, Blockchain and Evolution in Social Media to Build Trust and Prevent Fake News. In2018 Fourth International Conference on Advances in Computing, Communication \& Automation (ICACCA) 2018 Oct 26 (pp. 1-6). IEEE.

[21] Paul S, Joy JI, Ahmed S, Sarker S, Das AK. Fake News Detection in Social Media using Blockchain., 2019 7th International Conference on Smart Computing \& Communications (ICSCC).

[22] A. Campan, A. Cuzzocrea, and T.M. Truta, "Fighting fake news spread in online social networks: Actual trends and future research directions", 2017 IEEE International Conference on Big Data (Big Data), 2017.

[23] E. Mustafaraj and P. T. Metaxas, "The Fake News Spreading Plague: Was it Preventable?", Proceedings of the 2017 ACM on Web Science Conference, Troy, New York, USA, 2017.

[24] Singhania S, Fernandez N, Rao S. 3han: A deep neural network for fake news detection. In International Conference on Neural Information Processing 2017 Nov 14 (pp. 572-581). Springer, Cham.

[25] Ahmed H, Traore I, Saad S. Detection of online fake news using N-gram analysis and machine learning techniques. In International Conference on Intelligent, Secure, and Dependable Systems in Distributed and Cloud Environments 2017 Oct 25 (pp. 127-138). Springer, Cham.
[26] Hannah Rashkin, Eunsol Choi, Jin Yea Jang, Svitlana Volkova, Yejin Choi, "Truth of Varying Shades: Analyzing Language in Fake News and Political Fact-Checking" Conference on Empirical Methods in Natural Language Processing, September 7-11, 2017, Copenhagen, Denmark, 2017.

[27] Oshikawa R, Qian J, Wang WY. A survey on natural language processing for fake news detection. arXiv preprint arXiv:1811.00770. 2018 Nov 2.

[28] Gupta A, Kuru P. Credibiliumaragty ranking of tweets during high impact events. InProceedings of the 1 st workshop on privacy and security in online social media 2012 Apr 17 (p. 2). Acm.

[29] Melgy A, Ibrahim H, Torky M, " Recognizing and Stopping Rumors Patterns in Social Networks", Indian Journal of Science and Technology, 10 (28), 2017.

[30] "Your Guide to Good Journalism-New Trust" (2015),(Online), http://newstrust.net/.(Access, Oct 2019).

[31] J. Gentry "Package Twitter R", [Online] available: http://cran. rproject.org/web/packages/twitterR/twitterR.pdf , 2011, (Access 14 May. 2015).

[32] Xpertin, https://www.xpertin.com/en/pages/how_it_works (Access, 1 Nov. 2019).

[33] Kumar KK, Geethakumari G. Detecting misinformation in online social networks using cognitive psychology. Human-centric Computing and Information Sciences. 2014 Dec;4(1):14. 